\title{
Breakpoints on Chromosomes 9 and 22 in Philadelphia Chromosome-positive Chronic Myelogenous Leukemia (CML) \\ Amplification of Rearranged c-abl Oncogenes in CML Blast Crisis
}

Steven J. Collins

Department of Medicine, Veterans Administration Medical Center, Seattle, Washington 98108

\begin{abstract}
We surveyed 20 Philadelphia chromosome $\left(\mathrm{Ph}^{\mathbf{1}}\right)$ positive chronic myelogenous leukemia (CML) samples by Southern blot hybridization to determine the location of the breakpoints that occur on chromosomes 9 and 22 in the $\mathrm{Ph}^{1}$ translocation. Only 3 of 20 samples exhibited breakpoints on chromosome 9 within 18 kilobases (kb) of the $v$-abl homologous sequences. Mapping of these three chromosome 9 breakpoints indicates that each is at a separate location within this 18-kb region, indicating that there are no breakpoint "hot spots" in this area. In contrast, all 20 CML samples exhibited breaks on chromosome 22 within a 5.0kb Bgl II fragment that lies within the previously described breakpoint cluster region (bcr). Several patients with CML blast crisis exhibiting multiple $\mathrm{Ph}^{1}$ chromosomes/metaphase exhibited amplified and rearranged $c$-abl-related fragments. These additional $\mathbf{P h}^{1}$ chromosomes in blast crisis cells do not arise from a second, independent 9:22 translocation but rather result from a duplication of the preexisting $\mathrm{Ph}^{1}$ chromosome.
\end{abstract}

\section{Introduction}

Over $90 \%$ of cases of chronic myelogenous leukemia (CML) ${ }^{1}$ exhibit the Philadelphia chromosome $\left(\mathrm{Ph}^{1}\right)$ that results from a reciprocal translocation between chromosomes 9 and 22 . In this reciprocal exchange a portion of the $q$ arm of chromosome 22 is translocated to chromosome 9 to form $9 q^{+}$, while a small portion of the distal end of the $q$ arm of chromosome 9 harboring the $c$-abl oncogene is translocated to chromosome $22(1,2)$. Therefore, $\mathrm{Ph}^{1}$ is a deleted form of chromosome $22\left(22 \mathrm{q}^{-}\right)$which harbors the $c-a b l$ oncogene translocated from chromosome 9 (Fig. 1).

The $c-a b l$ oncogene is defined by virtue of its homology to $v-a b l$, the transforming sequences of the Abelson murine leukemia virus (3). The normal physiologic function of $c-a b l$ is presently unknown although its homologue $v-a b l$ is known to have tyrosine kinase activity (4). An abnormally large 8-kb ablrelated RNA transcript has been noted in CML cells $(5,6)$ and this aberrant message appears to be transcribed from the translocated $c$-abl oncogene on the $\mathrm{Ph}^{1}$. This abnormally large mRNA may code for $\mathrm{P} 210$, the abnormal-sized $a b l$-related protein product that has been noted recently in CML cells $(7,8)$. Thus the $c-a b l$ oncogene appears to be intimately related to the pathogenesis of CML.

Address reprint requests to Dr. Steven Collins, Fred Hutchinson Cancer Research Center, 1124 Columbia St., Seattle, WA 98104.

Received for publication 26 November 1985.

1. Abbreviations used in this paper: bcr, breakpoint cluster region; $\mathrm{CML}$, chronic myelogenous leukemia; $\mathbf{P h}^{1}$, Philadelphia chromosome.

The Journal of Clinical Investigation, Inc.

Volume 78, November 1986, 1392-1396
Initial reports have indicated that the breaks on chromosome 9 in CML occur at a variable distance from the $5^{\prime}$ end of $c-a b l$ (9). In contrast, chromosome 22 breaks appear to occur in a more restricted region termed the breakpoint cluster region (bcr) (10). However, more recent studies indicate that some $\mathrm{Ph}^{1}$-positive leukemic cells harbor chromosome 22 breaks outside the bcr $(11,12)$. To more thoroughly characterize the breakpoint locations on chromosomes 9 and 22, which are involved in the formation of $\mathrm{Ph}^{1}$, we studied $20 \mathrm{Ph}^{1}$-positive CML cells and cell lines using Southern blot hybridization with $c-a b l$ and bcr probes. During this study we noted several patients in CML blast crisis with multiple $\mathrm{Ph}^{1}$ chromosomes/metaphase who demonstrated amplified, rearranged $c$-abl-related fragments. We provide evidence that these additional $\mathrm{Ph}^{1}$ chromosomes in blast crisis cells arose from a duplication of a preexisting $\mathrm{Ph}^{1}$ chromosome rather than resulting from a second independent 9:22 translocation event.

\section{Methods}

Cells. The $\mathrm{Ph}^{1}$-positive CML cell line KCL-22 was cultured by means of standard techniques as referenced (13). The remaining cells were isolated from peripheral blood or bone marrow samples of CML patients being treated at the Seattle Veterans Administration Medical Center or the Fred Hutchinson Cancer Research Center. The presence of the $\mathbf{P h}^{1}$ was confirmed in clinical samples by standard cytogenetic analysis performed in the cytogenetic laboratories of the University of Washington Hospital and Fred Hutchinson Cancer Research Center. Leukocytes were isolated from patient samples using dextran sedimentation. The samples were diluted 1:1 in RPMI, and the red cells sedimented by incubation for $1 \mathrm{~h}$ in an equal volume of 3\% dextran T-500 at room temperature. The supernate cells were pelleted by centrifugation at $1,500 \mathrm{rpm}$, and the contaminating red cells in this pellet were subjected to hypotonic lysis in $0.2 \% \mathrm{NaCl}$, then an additional equal volume of $1.6 \% \mathrm{NaCl}$. Before DNA extraction, the cells were washed three times in plain RPMI.

Molecular probes. A molecular probe from the $5^{\prime}$ end of the $c$-abl oncogene was cloned from a genomic library constructed from the $\mathrm{K}$ 562 CML blast crisis cell line (14). DNA from this cell line was partially digested with Sau 3A, fractionated by sucrose density gradient centrifugation, and the 15-23-kb DNA fragments were isolated. This DNA was ligated with Bam-digested EMBL 3 phage vector DNA, and the ligation mixture was packaged in vitro. Approximately 500,000 recombinant clones were screened with a nick-translated 550-bp Hinc II-Sma I fragment derived from the pAB1 sub9 plasmid, which contains a $v$-abl insert (15). This 550-bp fragment represents the most $5^{\prime}$ sequences of $v$-abl. It was isolated from a polyacrylamide gel following endonuclease digestion of pAB1 sub9 (16). After screening, 10 positive clones were isolated and purified. One of these clones contained a 17-kb genomic insert that exhibited a restriction map similar to that of the previously published 5' end of $c-a b l(9)$. From this clone a 0.3-kb Hind III-Eco $\mathbf{R}_{\mathbf{1}}$ fragment, free of human repetitive sequences, was isolated and used as a molecular probe.

The ber probe consisted of a 1.2-kb Hind III-Bgl II fragment lying within the previously described bcr region on chromosome $22(10)$ and was purchased from Oncogene Science, Inc., Mineola, NY. 


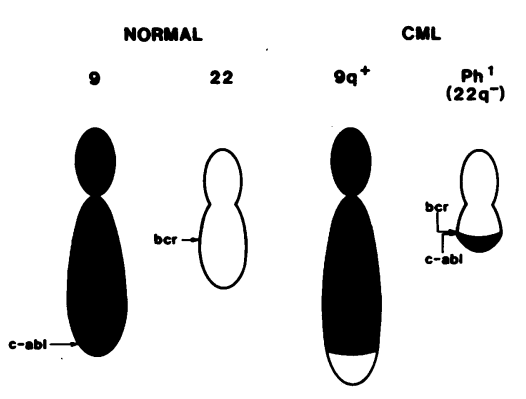

Figure 1. Genesis of $\mathrm{Ph}^{1}$ in chronic myelogenous leukemia. The $c-a b l$ oncogene is translocated from chromosome 9 to ber on chromosome 22 $\mathrm{q}^{-}$to form $\mathrm{Ph}^{1}$. The reciprocal translocation involves a portion of the $q$ arm of chromosome 22 moving to chromosome 9 to form $9 q^{+}$.

DNA extraction, Southern blot hybridization. DNA was extracted from the isolated leukocytes by digesting nuclei with protease $K$, then phenol/chloroform extracting and ethanol precipitating, as previously described (17). Standard techniques were used to perform restriction endonuclease digestion and Southern blotting. The blots were hybridized to nick translated probes and then washed under exactly the same conditions described previously (17).

\section{Results}

Localization of the breakpoints on chromosome 22. The Philadelphia chromosome results from a reciprocal translocation between chromosomes 9 and 22 (Fig. 1). Groffen et al. originally reported that 17 of $17 \mathrm{Ph}^{1}$-positive CML cells exhibited a breakpoint on chromosome 22 within a limited 5.8-kb bcr (10). In contrast, more recent reports indicate that some $\mathrm{Ph}^{1}$-positive patients may exhibit chromosome 22 breakpoints outside this bcr $(11,12)$. To examine this question, we analyzed $20 \mathrm{Ph}^{1}$ positive CML samples by Southern blot hybridization to determine the chromosome 22 breakpoint. We used a 1.2-kb Hind III-Bgl II bcr fragment which lies at the $3^{\prime}$ end of a $5.0-\mathrm{kb} \mathrm{Bgl}$ II fragment within the bcr as a molecular probe (Fig. 2). If chromosome breaks occur within this 5.0-kb Bgl II fragment, then

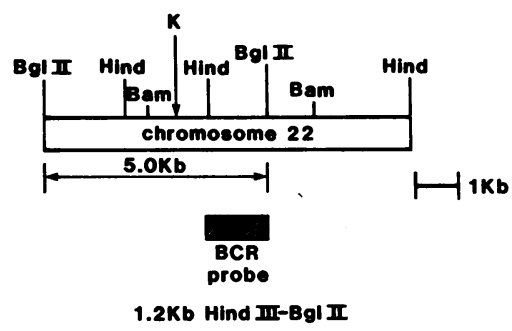

Figure 2. Restriction map of ber on chromosome 22 . The indicated ber probe is a $1.2-\mathrm{kb}$ Hind III-Bgl II fragment that hybridizes to a 5.0kb Bgl II germline fragment. The solid arrow depicts the approximate bcr breakpoint of CML patient $\mathrm{K}$. This restriction map is derived from reference 10 .

this probe, when hybridized to Southern blots of Bgl II digests of the CML DNA, should detect an extra fragment or fragments in addition to the germline $5.0-\mathrm{kb}$ Bgl II fragment. As shown in Fig. 3, all 20 of the CML samples exhibited an additional Bgl II fragment as well as the germline 5.0-kb Bgl II fragment. Thus, the breakpoint on chromosome 22 lies within this 5.0-kb Bgl II fragment in all 20 of these CML samples.

Localization of the breakpoint on chromosome 9. The c-abl oncogene is translocated from chromosome 9 to the Philadelphia chromosome in cases of CML. In contrast to the breakpoint on chromosome 22, the location of the breakpoint on chromosome 9 , while invariably occurring at the $5^{\prime}$ end of the $c$-abl gene, has been reported to be variable $(9,10)$. To access the breakpoint location on chromosome 9 in our 20 CML samples, we used a 0.3-kb Eco $R_{1}$-Hind III fragment that we cloned from a K-562 genomic EMBL 3 library. This library was constructed and screened as described in the Methods section. This subcloned 0.3-kb Eco $R_{1}$-Hind III fragment is free of repetitive sequences and lies within a 12-kb Bgl II as well as within a 15-kb Bam fragment which are both $5^{\prime}$ to the $v$-abl homologous sequences on chromosome 9 (Fig. 4). This probe therefore can detect breaks as far as $18 \mathrm{~kb}$ from the $v$-abl homologous sequences (Fig. 4).

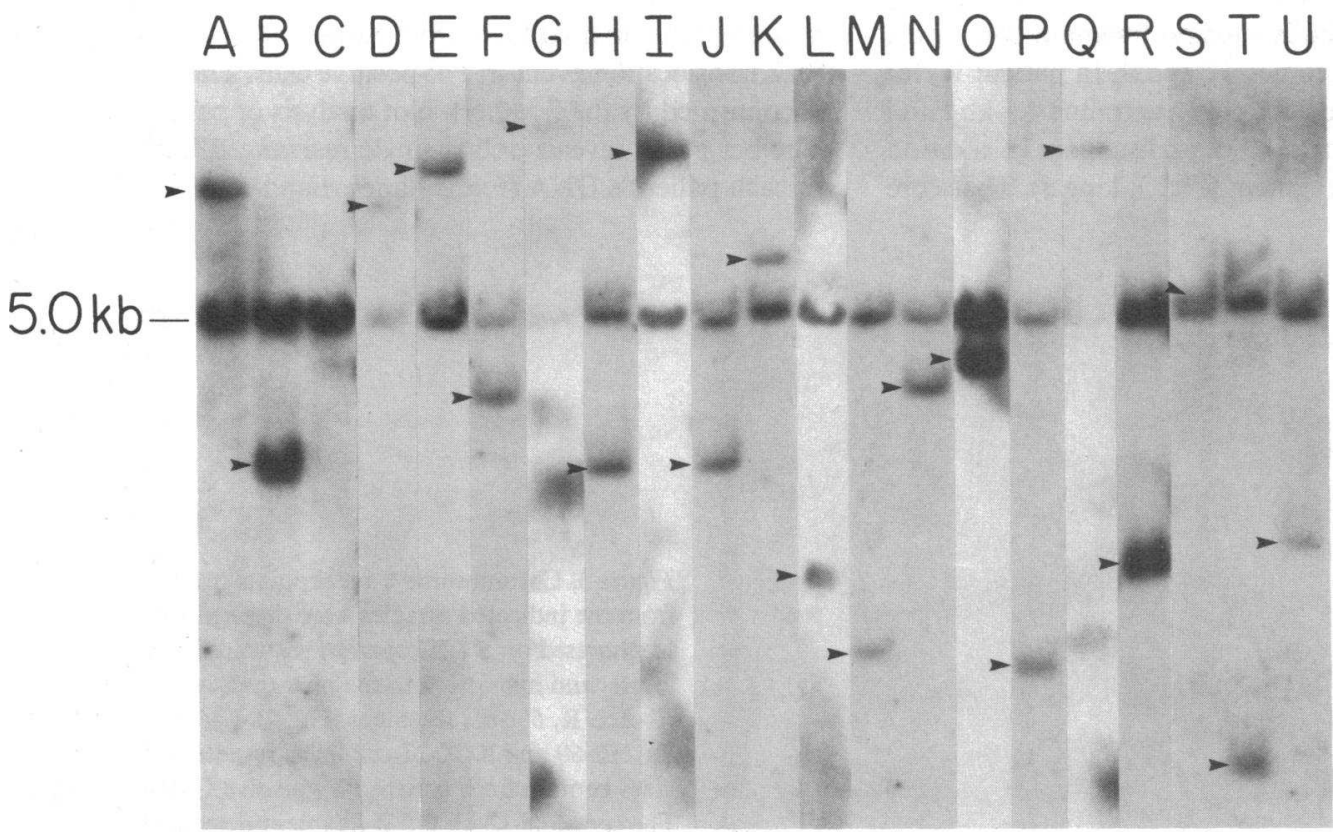

Figure 3. Chromosome 22 breakpoints in CML cells. DNA from the indicated samples were digested with Bgl II, electrophoresed on a $1.2 \%$ agarose gel, transferred to nitrocellulose, and hybridized to a nick-translated 1.2-kb Hind III-Bgl II ber probe (see Fig. 2). Lane $F$ contains the $\mathrm{Ph}^{1}$-positive CML blast crisis cell line KCL-22 DNA. Lane $C$ contains $\mathrm{Ph}^{1}$-negative $\mathrm{HL}$ 60 promyelocytic leukemia cell line DNA. All other samples represent fresh patient $\mathrm{Ph}^{1}$-positive CML cells. The germline 5.0-kb Bgl II fragment detected by the bcr probe is indicated. Arrows indicate additional bcr fragments detected by this probe.

"Smudges" seen in the lower molecular weight region of lanes $G$ and $Q$ were not present in other Southern blots of the same samples and most likely represent blot artifacts. 


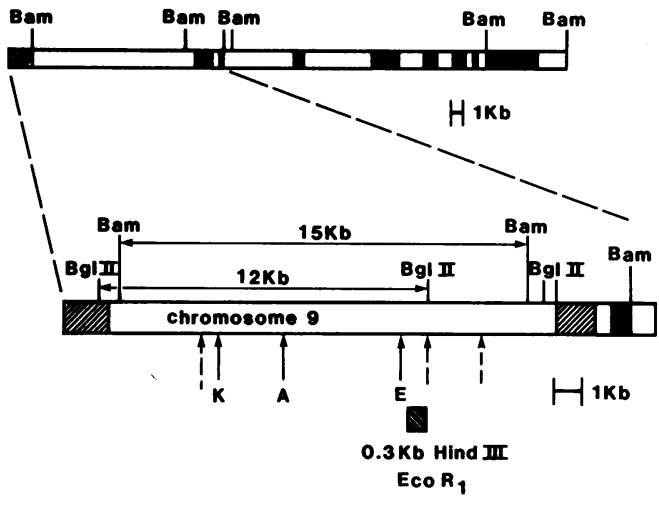

Figure 4. Breakpoints on chromosome 9 in CML cells. The top map depicts the $c-a b l$ locus on chromosome 9 . The bottom map represents the $5^{\prime}$ region of this locus. These maps are derived from references 3 , 9, and 19 and from our own mapping data. Exons are depicted by boxes. Solid boxes representing $v-a b l$ homologous exons, and hatched boxes represent additional exons that are unrelated to $v$-abl (19). Arrows depict the approximate breakpoint on chromosome 9 of various CML cells. Dashed arrows indicate previously localized CML breakpoints on chromosome 9 , as described in references 9 and 11 . Solid arrows represent chromosome 9 breakpoints in the CML patients $A$, $E$, and $K$, described in this work. The 0.3-kb Hind III-Eco $R_{1}$ fragment used as the $5^{\prime} c$-abl probe is indicated.

This probe was hybridized to Southern blots of Bam and Bgl II digests of genomic DNA from our 20 CML samples. 3 of the 20 samples revealed an extra Bgl II fragment in addition to the germline 12-kb Bgl II fragment, indicating a break within this fragment in these three CML cells (Fig. 5). This was confirmed by hybridizing the same probe to Southern blots of Bam digests of the CML samples. Only the three samples with an extra Bgl II fragment exhibited an extra Bam fragment (Fig. 6).

To further localize the chromosome 9 breakpoint region in these $3 \mathrm{CML}$ patients, we localized the breakpoint within the chromosome 22 bcr by restriction analysis and then determined the chromosome 9 breakpoint location from the size of the aberrant bcr Bgl II fragment, which is a fusion fragment containing sequences from both chromosomes 22 and 9 . In patient $\mathrm{K}$, for example, the bcr probe detected not only a germline $4.5-\mathrm{kb}$ Hind III fragment, but also an extra 7.5-kb Bam fragment in addition to the germline 3.5-kb Bam fragment (Fig. 7, lane 3). Therefore the breakpoint on chromosome 22 in patient $\mathrm{K}$ must lie in the 1.3-kb Bam-Hind III fragment within the bcr (Fig. 2, arrow) and is $1.3-2.6 \mathrm{~kb}$ from the $3^{\prime} \mathrm{Bgl}$ II site (Fig. 2). The aberrant Bgl II fragment detected by the bcr probe in patient $\mathrm{K}$ is $6 \mathrm{~kb}$ (Fig. 3) and represents a junction fragment with chromosome 9 sequences at its $5^{\prime}$ end and chromosome 22 sequences at its $3^{\prime}$ end (Fig. 8). Since this $9 q^{+}$junction fragment is $6 \mathrm{~kb}$, the distance from the $5^{\prime}$ end of this junction fragment to the chromosome 9 breakpoint must be 4.7-3.4 kb (Fig. 8). By similar reasoning we mapped the approximate breakpoint on chromosome 9 in patients $E$ and $A$. Unlike patient $K$, these patients exhibited germline fragments in both Hind III and Bam digests, indicated by the 1.2-kb Hind III-Bgl II bcr probe (Fig. 7). Therefore the chromosome 22 breakpoints in patients $\mathrm{A}$ and $\mathrm{E}$ must lie in the 2.4$\mathrm{kb}$ Bgl II-Bam fragment at the $5^{\prime}$ end of the bcr (Fig. 2) and therefore are located 2.6 to $5.0 \mathrm{~kb}$ from the $3^{\prime} \mathrm{Bgl} \mathrm{II}$ site. The $9 \mathrm{q}^{+} \mathrm{Bgl}$ II junction fragments detected by the bcr probe in patients $\mathrm{A}$ and $\mathrm{E}$ (Fig. 3) were 10.8 and $14.8 \mathrm{~kb}$, respectively. Therefore the distance from the chromosome 9-5' Bgl II end of this junction fragment to the breakpoint on chromosome 9 must be somewhere from 5.8 to $8.2 \mathrm{~kb}$ for patient $\mathrm{A}$ and $9.8-12.2 \mathrm{~kb}$ for patient $\mathrm{E}$. These approximate chromosome 9 breakpoint locations are noted by the solid arrows in Fig. 4.

Multiple $\mathrm{Ph}^{1}$ chromosomes in CML blast crisis: amplification of rearranged c-abl fragments. The evolution of CML from chronic phase to blast crisis is commonly associated with karyotypic changes, the most frequent of which is the development of two or more $\mathrm{Ph}^{1}$ chromosomes (18). It is uncertain whether these new $\mathrm{Ph}^{1}$ chromosomes arise from a new 9:22 translocation event or whether they represent duplication of the existing $\mathrm{Ph}^{1}$ chromosome. Karyotypic analysis of cells from patients $K$ and A, both of whom were in blast crisis, revealed the presence of two and three $\mathrm{Ph}^{1}$ chromosomes, respectively, per metaphase. In both these cells, the aberrant Bgl II fragment detected by the Hind III-Eco $R_{1} 5^{\prime} a b l$ probe appears to be amplified, since this probe hybridizes more intensely to the rearranged $\mathrm{Bgl}$ II fragment than to the germline fragment (Fig. 5, lanes $A$ and $K$ ). This amplification most likely results from a duplication (or triplication) of the original $\mathrm{Ph}^{1}$ chromosome rather than from a new 9:22 translocation event in $\mathrm{Ph}^{1}$-positive cells. This interpretation is confirmed by the Southern blot analysis of patients $A$ and $K$. The bcr probe reveals only a single rearranged $\mathrm{Bgl}$ II fragment in each patient's DNA (Fig. 3, lanes $A$ and $K$ ).

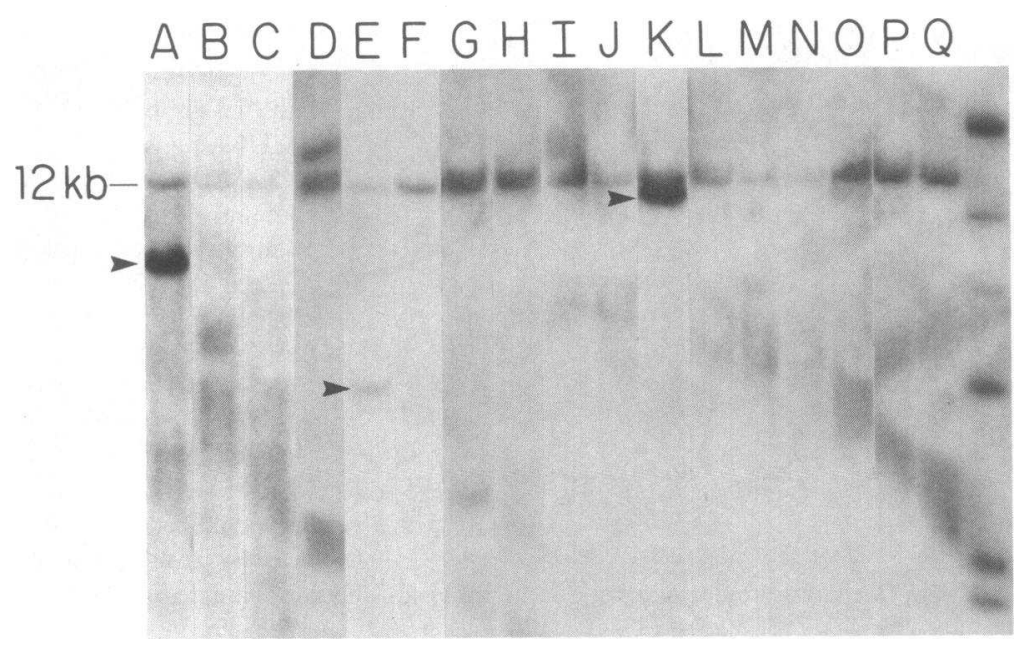

Figure 5. Chromosome 9 breakpoints in CML cells. DNA from the indicated samples were digested with Bgl II, electrophoresed on a $1.2 \%$ agarose gel, transferred to nitrocellulose, and hybridized to the nick-translated $0.3-\mathrm{kb}$ Hind III-Eco $\mathrm{R}_{1} 5^{\prime} c$ - $a b l$ fragment (Fig. 4). Lanes $C$ and $F$ are the HL-60 and KCL-22 cell lines, respectively. All other lanes contain fresh patient $\mathbf{P h}^{1}$-positive CML cell DNA. The germline 12-kb Bgl II fragment detected by this probe is indicated. Arrows indicate additional fragments detected by this probe in patients $A, E$, and $K$. 


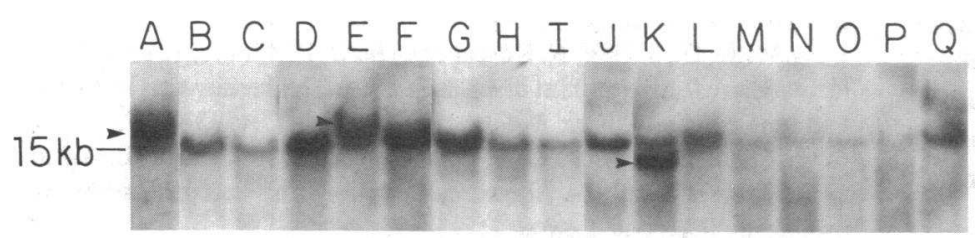

Figure 6. Chromosome 9 breakpoints in CML cells. The same samples indicated in Fig. 5 were digested with Bam $\mathrm{H}_{1}$, electrophoresed on a $1.2 \%$ agarose gel, transferred to nitrocellulose, and hybridized to the nick-translated 0.3-kb Hind IIIEco $R_{1} 5^{\prime} c$-abl fragment (Fig. 4). The germline 15-kb Bam fragment detected by this probe is indicated. Arrows indicate additional fragments detected by this probe in patients A, E, and $K$.

\section{Discussion}

We undertook this survey of $20 \mathrm{Ph}^{1}$-positive $\mathrm{CML}$ cells and cell lines in an attempt to localize the breakpoints on chromosomes 9 and 22 that are involved in the generation of $\mathrm{Ph}^{1}$. We were particularly interested in determining the breakpoint locations on chromosome 9 , since initial reports indicated that these breaks occurred at a variable distance from the $5^{\prime}$ end of the $c$ - $a b l$ oncogene $(9,10)$. Using a probe at the $5^{\prime}$ end of $c$-abl, which can determine breakpoints within $18 \mathrm{~kb}$ of the $v$-abl homologous sequences when hybridized to the appropriate Southern blots, we found breaks in this region in only 3 of the $20 \mathrm{CML}$ samples analyzed. Using similar molecular probes, Heisterkamp et al. (9) initially described breaks in this region in one of three $\mathrm{Ph}^{1}$ positive CML samples while Leibowitz et al. (11) more recently described breaks in this location in only 2 of 15 CML samples. Together with our own findings, these data indicate that only $\sim 15 \%$ (6 of 38) of CML samples exhibit breaks on chromosome 9 within $18 \mathrm{~kb}$ of the $v$-abl homologous sequences. Most chromosome 9 breaks presumably occur at a distance more $5^{\prime}$ to this region.

Canaani has recently mapped several exons at the 5 end of $c$ - $a b l$ which are not homologous to $v$ - $a b l$ (Fig. 4, hatched boxes, reference 19 ). Conceivably there may be other more $5^{\prime} c$ - $a b l$ exons that have not yet been mapped. The three chromosome 9 breakpoints we have mapped in this work, as well as the three others reported to date, all lie within a large intron between the first two of these recently described exons (Fig. 4). As is evident from Fig. 4, the reported chromosome 9 breakpoints are dispensed widely throughout this large intron, and there is no particular "hot spot" for chromosome breakage in this region.

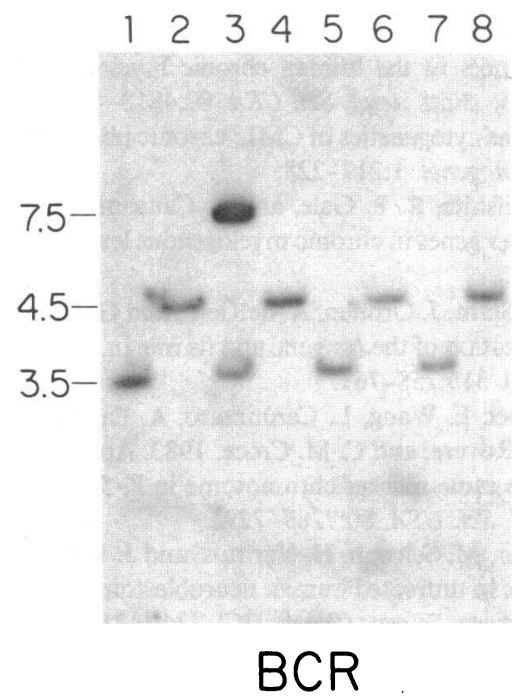

Figure 7. Localization of the chromosome 22 breakpoint in CML patients $A, E$, and $K$. DNA samples were digested with specific restriction enzymes, electrophoresed in $1.2 \%$ agarose, transferred to nitrocellulose, and hybridized to a nick-translated 1.2-kb Hind III-

Bgl II bcr probe. Lanes

1 and 2 are HL-60 DNA representing germline bcr region. Lanes 3 and 4 are patient $K$ DNA. Lanes 5 and 6 are patient A DNA and lanes 7 and 8 are patient $E$ DNA. Lanes $1,3,5$, and 7 are Bam $\mathrm{H}_{1}$ digests. Lanes 2, 4, 6, and 8 are Hind III digests.

In contrast to the breaks on chromosome 9 , the breaks on chromosome 22 have been reported to be confined to a relatively small locus, the ber (10). This $\sim 6-\mathrm{kb}$ region appears to lie within a much larger coding region of at least $45 \mathrm{~kb}(20)$. The function of the gene product of this region is unknown, but Canaani has recently demonstrated by cDNA cloning and DNA sequencing that the aberrantly large 8-kb RNA transcript observed in CML cells is a fusion transcript between bcr coding sequences on chromosome 22 and the translocated $c$-abl sequences from chromosome 9 (19). This aberrantly large RNA transcript may code for $\mathrm{P} 210$, an abnormally large $a b l$-related protein product that has been observed in CML cells $(7,8)$. Groffen et al. originally noted 17 of $17 \mathrm{Ph}^{1}$-positive CML patients with chromosome 22 breaks in the bcr (10). However, several recent reports have described $\mathrm{Ph}^{1}$-positive samples where the chromosome 22 breakpoint apparently lies outside the bcr $(11,12)$. In our survey, we found that all 20 of our $\mathrm{Ph}^{1}$-positive CML samples exhibited breaks in a $5.0-\mathrm{kb}$ Bgl II fragment within the bcr region. These findings confirm the original observations of Groffen and emphasize the usefulness of ber probes in identifying by restriction analysis CML cells harboring $\mathrm{Ph}^{1}$. Indeed, we are currently using this probe to identify residual or recurrent disease among $\mathbf{P h}^{1}$ positive CML patients who have undergone bone marrow transplantation.

The bcr probe should be particularly useful in these bone marrow transplant studies because it provides a unique marker for individual $\mathrm{Ph}^{1}$ chromosomes. Since the breakpoints on chromosomes 9 and 22 are variable for individual $\mathrm{Ph}^{1}$ chromosomes, the aberrant restriction fragment detected by the bcr probe will vary in size in different $\mathrm{Ph}^{1}$-positive $\mathrm{CML}$ samples. (Note that all 20 of the aberrant bcr fragments displayed by the $\mathrm{Ph}^{1}$-positive CML cells in Fig. 3 are different sizes.) This probe, when used to analyze CML transplant patients who have relapsed, can distinguish patients who relapse with a $\mathrm{Ph}^{1}$ chromosome identical to the one present before transplant from those who relapse with a $\mathrm{Ph}^{1}$ arising de novo from a new translocation event.

The development of multiple $\mathrm{Ph}^{1}$ chromosomes is the most frequent karyotypic abnormality that occurs as $\mathrm{CML}$ evolves from chronic to blast crisis phase. It is unclear whether these additional $\mathrm{Ph}^{1}$ chromosomes are a consequence of duplication of a preexisting $\mathrm{Ph}^{1}$ or whether they arise as a result of a new 9:22 translocation event. We were able to approach this question

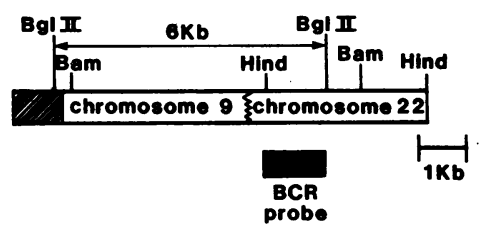

Figure 8. Map of the $9 \mathrm{q}^{+}$ breakpoint region in patient K. The aberrant $6.0-\mathrm{kb} \mathrm{Bgl}$ II fragment detected by the bcr probe is a fusion fragment with the $5^{\prime}$ Bgl II site derived from chromosome 9 and the $3^{\prime} \mathrm{Bgl}$ II site derived from chromosome 22 . 
in our survey, since two of the three CML samples exhibiting breaks relatively close to the $v-a b l$ homologous sequences were blast crisis cells harboring two and three $\mathbf{P h}^{1}$ chromosomes/ metaphase, respectively. Both these samples exhibit amplification of the rearranged $5^{\prime} c$-abl fragment when compared with the germline fragment (Fig. 5, lanes $A$ and $K$ ). Since this rearranged fragment is present on the $\mathrm{Ph}^{1}$, the presence of multiple $\mathrm{Ph}^{1}$ chromosomes harboring identically rearranged fragments is the most likely explanation for the $c$-abl fragment amplification. These extra $\mathrm{Ph}^{1}$ chromosomes most likely arose from a duplication of preexisting $\mathrm{Ph}^{1}$ chromosomes rather than from a new 9:22 translocation event. Southern blots of DNA digests using the bcr probe confirm this interpretation. If the new $\mathrm{Ph}^{1}$ chromosome arose from a second independent 9:22 translocation event, then a second rearranged bcr fragment should be noted. However, only a single rearranged bcr fragment is noted in the Southern blots from these samples (Fig. 3, lanes $A$ and $K$ ), thus confirming that the new $\mathrm{Ph}^{1}$ chromosomes arose from a duplication (or triplication) of the initial $\mathrm{Ph}^{1}$.

The presence of these multiple $\mathrm{Ph}^{1}$ chromosomes in $\mathrm{CML}$ blast crisis vs. chronic phase cells and the consequent amplification of the translocated $c-a b l$ oncogene emphasize the important role this oncogene may play in the inevitable progression of CML from chronic phase to blast crisis. The data presented here provide the first direct evidence for amplification of the $c$-abl oncogene in fresh CML blast crisis cells. We have previously noted a four- to eight-fold amplification of the $c$-abl oncogene in the cultured CML blast crisis cell line K-562 (17) (Interestingly, unlike these fresh CML cells, the K-562 cell line lacks multiple $\mathrm{Ph}^{1}$ chromosomes, but the $c-a b l$ oncogene appears to be tandemly amplified on an aberrant marker chromosome [21].) We have also noted increased RNA expression of the $c$-abl oncogene in CML blast crisis vs. chronic phase cells (6). Oncogene amplification and enhanced expression may play an important role in the progression of certain human malignancies. For instance, amplification and enhanced expression of the $N-m v c$ oncogene has recently been related to progression of childhood neuroblastoma $(22,23)$. Whether the enhanced expression of $c$ - $a b l$ is the major event in the evolution of CML from chronic phase to blast crisis or whether other important genetic events are involved in the progression of CML is presently unknown.

\section{Acknowledgments}

I wish to thank LeMoyne Mueller and Monique Howard for excellent technical assistance. I am grateful to Dr. Jean Wang for providing the $v$-abl pAB1 sub9 plasmid. This work was supported by the Veterans Administration and by National Cancer Institute grant CA 40728-01. Dr. Collins is a Scholar of the Leukemia Society of America.

\section{References}

1. Rowley, J. D. 1973. A new consistent chromosomal abnormality in chronic myelogenous leukemia identified by quinacrine fluorescence and Giemsa staining. Nature (Lond.). 243:290-293.

2. deKlein, A., A. Geurts van Kessel, G. Grosveld, C. Bartram, A. Hagemeijer, D. Bootsma, N. K. Spurr, N. Heisterkamp, J. Groffen, and J. R. Stephenson. 1982. A cellular oncogene is translocated to the Philadelphia chromosome in chronic myelocytic leukemia. Nature (Lond.). 300:765-767.
3. Heisterkamp, N., J. Groffen, and J. R. Stephenson. 1983. The human v-abl cellular homologue. J. Mol. Appl. Genet. 2:57-68.

4. Witte, D., S. Goff, N. Rosenberg, and D. Baltimore. 1980. A transformation defective mutant of Abelson murine leukemia virus lacks protein kinase activity. Proc. Natl. Acad. Sci. USA. 77:4993-4997.

5. Canaani, E., R. P. Gale, D. Steiner-Saltz, A. Berrebi, E. Aghai, and E. Januszewicz. 1984. Altered transcription of an oncogene in chronic myelocytic leukemia. Lancet. 1:593-595.

6. Collins, S. J., I. Kubonishi, I. Miyoshi, and M. T. Groudine. 1984. Altered transcription of the $c-a b l$ oncogene in K-562 and other chronic myelogenous leukemia cells. Science (Wash. DC). 225:72-75.

7. Konopka, J. B., S. M. Watanabe, and O. N. Witte. 1984. An alteration of the human $c-a b l$ protein in K-562 leukemia cells unmasks associated tyrosine kinase activity. Cell. 37:1035-1042.

8. Konopka, J. B., S. M. Watanabe, J. W. Singer, S. J. Collins, and O. N. Witte. 1985. Cell lines and clinical isolates derived from $\mathrm{Ph}^{1}$ positive chronic myelogenous leukemia patients express $c-a b l$ proteins with a common structural alteration. Proc. Natl. Acad. Sci. USA. 82: 1810-1814.

9. Heisterkamp, N., J. R. Stephenson, J. Groffen, P. F. Hansen, A. deKlein, C. R. Bartram, and G. Grosveld. 1983. Localization of the $c$-abl oncogene adjacent to a translocation breakpoint in chronic myelocytic leukemia. Nature (Lond.). 306:239-242.

10. Groffen, J., J. R. Stephenson, N. Heisterkamp, A. deKlein, C. R. Bartram, and G. Grosveld. 1984. Philadelphia chromosomal breakpoints are clustered within a limited region, $b c r$, on chromosome 22. Cell. 36:93-99.

11. Leibowitz, D., K. Schaefer-Rego, D. Popenoe, J. G. Mears, and A. Bank. 1985. Variable breakpoints on the Philadelphia chromosome in chronic myelogenous leukemia. Blood. 66:243-245.

12. Rodenhuis, S., L. Smets, R. Slater, H. Behrendt, and A. Veerman. 1985. Distinguishing the Philadelphia chromosome of acute lymphoblastic leukemia from its counterpart in chronic myelogenous leukemia. N. Engl. J. Med. 313:51.

13. Kubonishi, I., and I. Miyoshi. 1983. Establishment of $\mathrm{Ph}^{1}$ chromosome positive cell line from chronic myelogenous leukemia in blast crisis. Int. J. Cell Cloning. 1:105-117.

14. Lozzio, C. B., and B. B. Lozzio. 1975. Human chronic myelogenous leukemia cell line with positive Philadelphia chromosome. Blood. 45:321-334.

15. Wang, J., and D. Baltimore. 1983. Cellular RNA homologous to the Abelson murine leukemia virus transforming gene: expression and relationship to the viral sequence. Mol. Cell. Biol. 3:773-779.

16. Maniatis, T., E. Fritsch, and J. Sambrook. 1983. Molecular Cloning, A Laboratory Manual. Cold Spring Harbor Laboratory, Cold Spring Harbor, NY. 178.

17. Collins, S. J., and M. T. Groudine. 1983. Rearrangement and amplification of $c$ - $a b l$ sequences in the human chronic myelogenous leukemia cell line K-562. Proc. Natl. Acad. Sci. USA. 80:4813-4817.

18. Sandberg, A. 1980. The cytogenetics of CML: chronic phase and blast crisis. Cancer Genet. Cytogenet. 1:217-228.

19. Shtivelman, E., B. Lifshitz, R. P. Gale, and E. Canaani. 1985. Fused transcripts of $a b l$ and $b c r$ genes in chronic myelogenous leukemia. Nature (Lond.). 315:758-761.

20. Heisterkamp, N., K. Stam, J. Groffen, A. deKlein, and G. Grosveld. 1985. Structural organization of the bcr gene and its role in the $\mathrm{Ph}^{1}$ translocation. Nature (Lond.). 315:758-761.

21. Selden, J., B. Emanuel, E. Wang, L. Cannizzaro, A. Palumbo, J. Erikson, P. C. Nowell, G. Rovera, and C. M. Croce. 1983. Amplified $C_{\lambda}$ and $c-a b l$ genes are on the same marker chromosome in K-562 leukemia cells. Proc. Natl. Acad. Sci. USA. 80:7289-7292.

22. Brodeur, G., R. Seeger, M. Schwab, H. Varmus, and J. Bishop. 1984. Amplification of $N$-myc in untreated human neuroblastomas correlates with advanced disease stage. Science (Wash. DC). 224:1121-1124.

23. Seeger, R., G. Brodeur, H. Sather, A. Dalton, S. Siegel, K. Wong, and D. Hammond. 1985. Association of multiple copies of the $N-m y c$ oncogene with rapid progression of neuroblastomas. N. Engl. J. Med. 311:231-235. 